\title{
Esophageal achalasia of unknown etiology in children
}

\author{
Pedro M. Fernandez, ${ }^{1}$ Luiz A.G. Lucio, ${ }^{2}$ Fabíola Pollachi²
}

\begin{abstract}
Objective: To report a case of a 9-year-old female presented with esophageal achalasia and approached with surgery. The authors discuss the treatment and make a literature review on the topic.

Description: Childhood esophageal achalasia is an unusual disease, often with unknown etiology. The main symptoms are esophageal vomits, dysphagia and weight loss. The diagnosis can be made by esophagogram and endoscopy, but the main examination is the esophageal manometry. Even though the surgical approach is a wellestablished therapy, some alternative treatments have been used, such as the endoscopy balloon dilatation and the use of botulinum toxin.

Comments: Esophageal achalasia is a rare disease in childhood, with unknown etiology. The presentation may be confused with gastroeshophageal reflux, sometimes causing a diagnosis delay. The surgical approach, as well as an antireflux procedure, is the treatment of choice.
\end{abstract}

J Pediatr (Rio J). 2004;80(6):523-6: Dilated esophagus, gastroeshophageal reflux, childhood, esophagomyotomy, pneumatic balloon dilatation, botulinum toxin.

\section{Introduction}

Esophageal achalasia of unknown etiology is a rare disease, affecting $1: 100,000$ of the general population and just 4 to $5 \%$ of these cases are described in children. ${ }^{1}$ Achalasia involves a failure of the lower esophageal sphincter (LES) in conjunction with reduced motility in esophagus itself. The passage of food via the esophagogastric junction is extremely difficult without true organic stenosis or extrinsic compression.

The symptom spectrum is wide, varying from regurgitation to recurrent aspiration pneumonia. Older children describe symptoms of progressive dysphagia. For these reasons the disease is often confused with

1. Adjunct professor. Chief of the Service of Pediatric Surgery, Faculdade de Medicina do ABC, Santo André, SP, Brazil.

2. Assistant physician, Service of Pediatric Surgery, Faculdade de Medicina do ABC, Santo André, SP, Brazil.

Manuscript received Feb 13 2004, accepted for publication Jun 092004.

Suggested citation: Fernandez PM, Lucio LA, Pollachi F. Esophageal achalasia of unknown etiology in children. J Pediatr (Rio J). 2004;80:523-6. gastroesophageal reflux disease (GERD), delaying correct diagnosis. 2,3

In response to the rarity of the case and the diagnostic difficulties the authors describe a case of childhood esophageal achalasia of unknown etiology.

\section{Case description}

A female patient, referred from the Centro Hospitalar de Santo André (C.H.M.S.A.), at 9 years of age, to the Pediatric Surgery Service at the ABC Medical Faculty, São Bernardo do Campo Teaching Hospital, complaining of vomiting for 2 months, immediately after meals, in moderate quantities, characteristic of the esophagus, of a pale color with recently ingested food clearly recognizable and without prior nausea. Vomiting was daily with a frequency of once a day, with 2 to 3 day periods of remission, although during the 15 days prior to the consultation there had been no more periods of remission and episodes had increased in frequency to two or three times a day. These symptoms were accompanied by weight loss of $10 \mathrm{~kg}$ over the period, despite the patient, at the time of admission, being on the 50th percentile for 
weight and height. The patient denied any dysphagia, odynophagia, nocturnal regurgitation or abdominal pains. Personal history included a viral infection of the upper airway for the previous 3 months accompanied by bronchospasm, 1 month before the onset of symptoms.

The esophagram revealed tapering of the esophagus close to the cardia, obstructing the passage of the contrast for 30 minutes after its ingestion (Figure 1). There was no gastroesophageal reflux and esophagus-esophageal reflux was present as were tertiary contractions, thus ruling out a hypothesis of GERD.

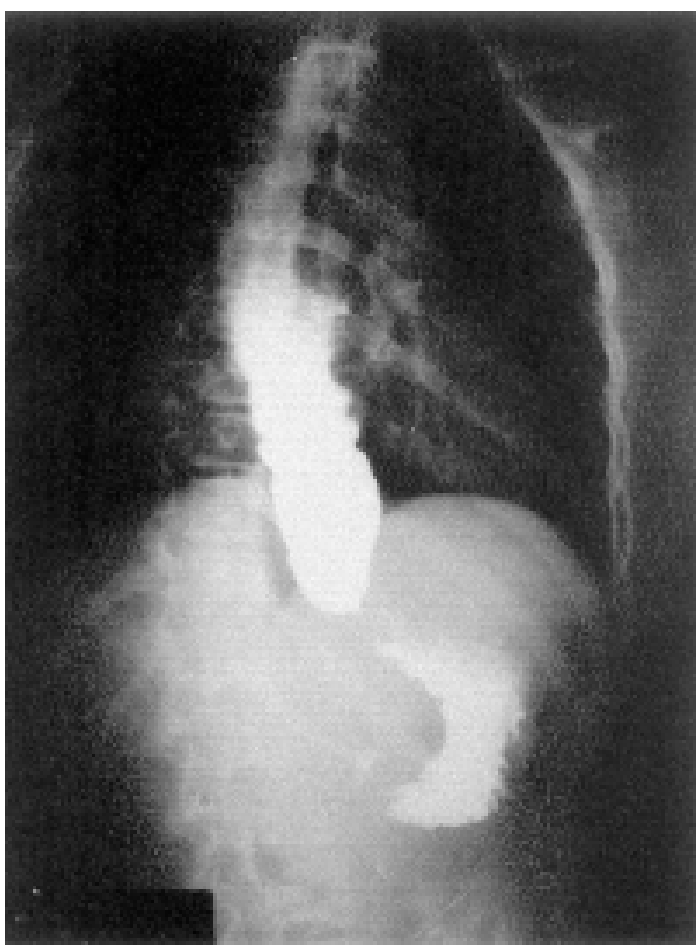

Figure 1 - Esophagram revealed tapering of the esophagus close to the cardia, obstructing the passage of the contrast, dilatation of the esophageal body and presence of air-fluid level
During upper digestive endoscopy, the endoscope encountered resistance close to the cardia and a biopsy taken endoscopically from the esophagus underwent anatomopathological examination, demonstrating chronic esophagitis with acanthosis. Esophageal electromanometry found that LES relaxation was absent after all deglutitions, in addition to increased basal pressure, compatible with achalasia esophageal (Figure 2).

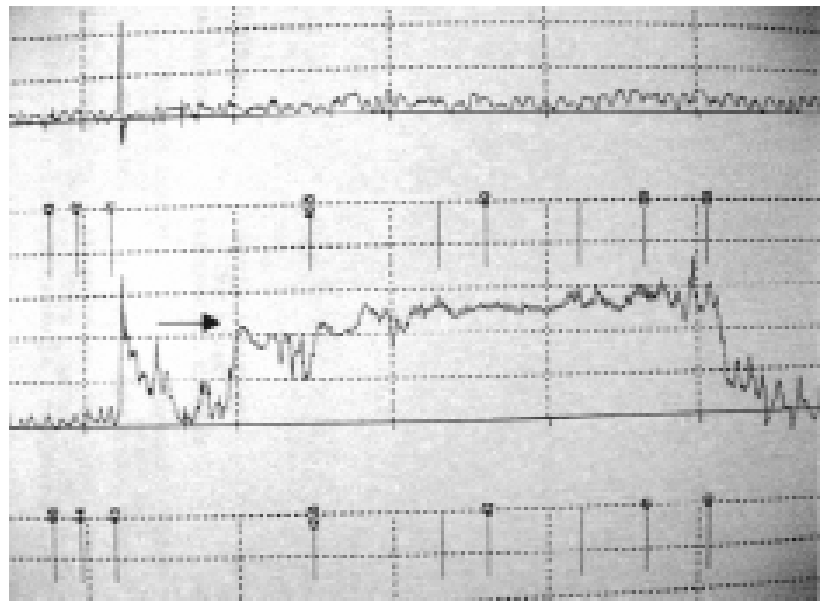

Figure 2 - Esophageal electromanometry showing absence of LES relaxation after deglutitions (arrow)

Once a diagnosis of esophageal achalasia had been made the child was subjected to cardiomyomectomy by laparotomy, and an anterior anti-reflux valve created (Dor Fundoplication), with no intra-operative or post-operative complications (Figure 3), being discharged on the fourth day after the operation. The anatomopathological study of the excised section demonstrated the presence of neural threads and an absence of intramural ganglion cells.

Currently, during the patient's second post-operative year, she continues under outpatients observation, is asymptomatic and is gaining weight. An esophagram and an upper digestive endoscopy were performed with normal results.

\section{Discussion}

Esophageal achalasia is a failure of the coordinated muscle relaxation mechanism of the LES, after a peristaltic contraction of the esophageal body, stimulated by a deglutition. ${ }^{2}$ The etiology of the disease is unknown and it is rare during childhood, there is no family association and it affects more male children (6:1) than female, while in the case described above the patient was female. ${ }^{2-5}$

The main theories that have been developed to explain the onset of this disease are: 1) a primary neurogenic abnormality with a failure of the inhibitory nerves and progressive degeneration of ganglion cells; 2) an acquired deficiency of the myenteric plexus ganglion cells, secondary to GERD, Chagas disease, or viral process. ${ }^{1,4}$

Clinical presentation varies from regurgitation of ingested food, described as vomiting immediately after meals, (associated or not with stasis, a deficit in pondero-statural growth or weight loss, repeated aspiration pneumonia, and, in older children, complaints of dysphagia. 1-3,6,7 Because of these symptoms and because it is related to GERD, diagnoses of achalasia of the esophagus are often underestimated. With respect of the present case we observed regurgitation and weight loss. 


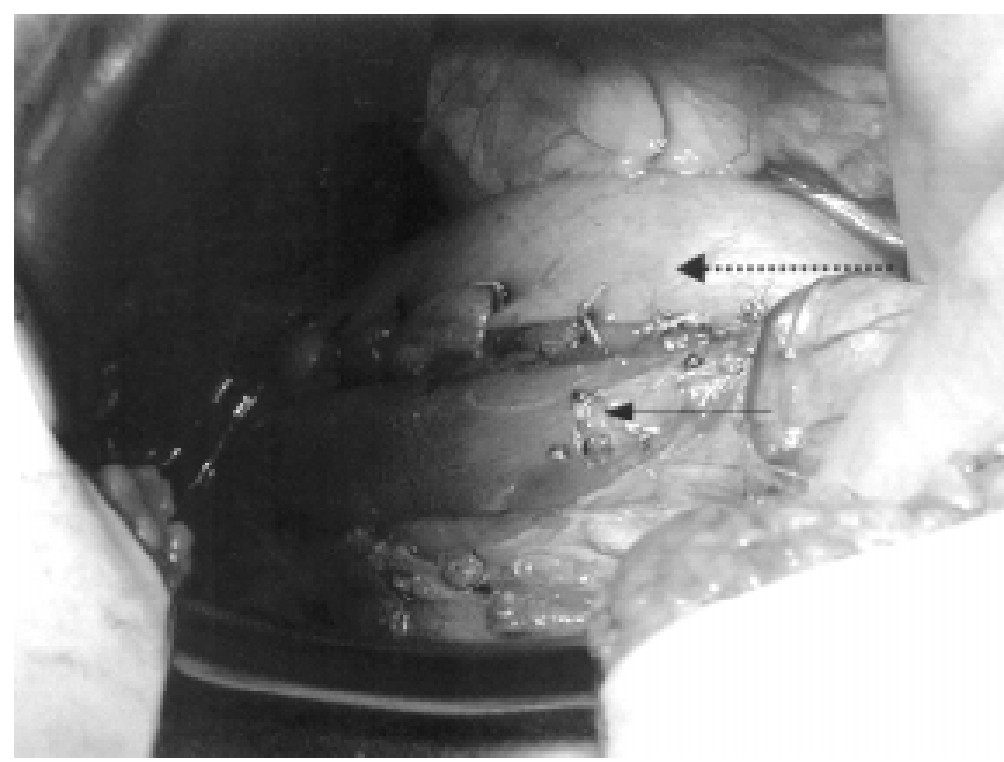

Figure 3 - Photography of the suture of anterior anti-reflux valve on the cardiomyomectomy area (arrow) partially covered by gastric fundus (hatched arrow)

The first diagnostic test is an esophagram. In the case described here, the distal third of the esophagus was observed to be tapering (the "bird's beak" appearance). In the dilated section, tertiary contractions of the esophageal body are found together with an air-fluid level, causing the observed difficulties in the passage of the contrast from the esophagus to the stomach (Figure 1), in common with reports in the literature. ${ }^{2-6}$

During endoscopic examination the endoscope encountered resistance to its progress, ${ }^{1-3,6}$ also in agreement with published literature. This examination is of extreme importance to confirm the presence of esophagitis and other types of injury present with GERD, such as esophageal stenosis. These injuries can be associated with achalasia. In our patient, we observed acanthosis and chronic esophagitis endoscopically. In addition to allowing a biopsy to be taken of the esophagus, endoscopy can also be used as a treatment method - as we will explain below.

Currently the examination of choice to confirm the presence of achalasia is esophageal manometry, ${ }^{7,8}$ in which increased LES basal pressure will be observed, accompanied by incomplete or absent LES relaxation after deglutition, associated with a lack of peristaltic coordination of the esophageal body during deglutition, as was the case with the patient in question. $2,7,8$ Scintillographic studies and esophageal $\mathrm{pH}$ tests are useful to diagnose or rule out GERD in association. These tests were not performed for the case described here. ${ }^{2-4}$
Classically, esophageal dilatation and esophageal myotomy by laparotomy has been the treatment of choice for the last 40 years. ${ }^{1,4}$ Nowadays there are other treatment options, such as medication-based therapy with calcium channel blockers, which act to relax the LES. This treatment has not been used for children, and its use on adults has not been effective. ${ }^{1,4}$ There is also the possibility of injecting botulinum toxin directly onto the LES. ${ }^{4}$ This treatment has been reserved for cases in which there is no possibility of a surgical solution and offers a short-term effect which needs to be repeated at ever-decreasing intervals. It has also been observed that its use can lead to the progressive destruction of the esophagus wall, in addition to making surgical procedures more difficult, ${ }^{4}$ for which reason we did not employ this type of procedure.

Pneumatic balloon dilatation has been defended by some authors, 1,9 but it is known that the effects are transitory and that a majority of patients present symptomatic relapses which may lead to GERD, in addition to the significant risk of esophageal perforation during dilatation. 4,6,9,10

The classical surgical approach consists of performing myotomy of the esophagus and cardia, while some authors suggest a variation of the technique, removing a strip of muscle tissue (myomectomy). This extends from the LES to 2 or $3 \mathrm{~cm}$ above the cardia, generally associated with anti-reflux procedures, and is done with a view to the fact that cardiomyomectomy alone favors gastroesophageal 
reflux. 2,6 These children exhibit good postoperative results, and certain series have demonstrated satisfactory results over long-term follow-up. ${ }^{11-13}$ The case described here is currently being followed-up in outpatients, eight months after the procedure and has remained asymptomatic to date, with a good level of dietary acceptance and ponderostatural gains.

During recent years laparoscopy has made surgical treatment of achalasia of the esophagus less invasive. The approach can be either abdominal or thoracic. The transthoracic approach with video allows the myotomy to be made with sufficient length, but does not allow the antireflux procedure to be performed. Postoperative $\mathrm{pH}$ testing reveals that transthoracic myotomies present a $60 \%$ level of gastroesophageal reflux complications. 4,10

The abdominal laparoscopic approach allows the myomectomy to be performed in association with the antireflux procedure and, of these, the one that has greatest acceptance is anterior Dor Fundoplication. This technique consists of re-covering the exposed esophageal mucosa with gastric fundus using an anterior route. In this manner later occurrence of GERD is avoided in addition to the exposed mucosa being protected. 4,10,12,13

According to certain authors, the so-called minimally invasive surgery (laparoscopic) does not just represent the most effective treatment for the disease, but also reduces hospitalization times (an average of 2 to 3 days) and hospital costs, allowing the child to return more quickly to their normal activities. ${ }^{10,14}$ It does, however, require specialized material and professionals for its use to be justifiable. In our country only a few services have this technology available and its use is thereby limited for children.

Postoperative manometric studies reveal that a few patients have a partial recuperation of esophageal peristalsis, being capable of generating the progressive waves, but not with enough force to be effective, leading to the belief that achalasia is not simply a failure of LES relaxation, but also involves generalized esophageal dysmotility. ${ }^{8}$

In the case described here we opted for Heller cardiomyomectomy a via laparotomy, with anterior Dor fundoplication, achieving good postoperative progress and hospital discharge in 4 days, within the expectations described in the literature, and with no sign of relapse to date.

\section{Conclusions}

Esophageal achalasia is a rare disease in children and its origin is generally indeterminable. The sufferer's symptomology may suggest gastroesophageal reflux, resulting in unsuccessful treatment. An esophagram and, more precisely, esophageal manometry can confirm diagnosis. Heller Cardiomyotomy is the treatment modality of choice at many services and can be associated with an anti-reflux valve, which was the choice made in the case described here and which resulted in good clinical progress.

\section{References}

1. Hamza AF, Awad HA, Hussein O. Cardiac Achalasia in Children. Dilatation or Surgery? Eur J Pediatr Surg. 1999;9:299-302

2. Jolley SG, Baron HI. Disorders of esophageal function. In: O'Neill JA. Pediatric Surgery. 5th ed. Mosby; 2000.vol 1. p. 9971007.

3. Ashcraft KW. The esophagus. In: O'Neill JA. Pediatric Surgery. 5th ed. Mosby; 2000. p. 325-47.

4. Patti MG, Albanese CT, Holcomb III GW, Molena D, Fisichella PM, Perretta S, et al. Laparoscopic Heller myotomi and Dor fundoplication for esophageal achalasia in children. J Pediatr Surg. 2001;36:1248-51.

5. Porras G, Hernández MH, Izundegui G, Porras JD. Selecting the surgical procedure for simple and complicated esophageal achalasia in children. J Pediatr Surg. 1997;32:1595-7.

6. Nakajima K, Wasa M, Kawahara H, Soh H, Taniguchi E, Ohashi $\mathrm{S}$, et al. Laparoscopic esophagomiotomy with Dor anterior fundoplicacion in a child with achalasia. Surg Laparosc Endosc Percutan Tech. 1999;10:236-9.

7. Chelimsky G, Hupertz V, Blanchard T. Manometric progression of achalasia. J Pediatr Gastroenterol Nutr. 2000;31:303-6.

8. Tovar JA, Prieto G, Molina M, Arana J. Esophageal function in achalasia: preoperative and postoperative manometric studies. J Pediatr Surg.1998;33:834-8.

9. Lan LC, Wong KK, Lin SC, Sprigg A, Clarke S, Johnson PR, et al. Endoscopic balloon dilatation of eshophageal strictures in infants and children: 17 years' experience anand a literature review. J Pediatr Surg. 2003;38:1712-5.

10. Rothenberg SS, Patrick DA, Bealer JF, Chang JHT. Evaluation of minimaly invasive approaches to achalasia in children. J Pediatr Surg. 2001;36:808-10.

11. Stiff GM, Khan R, Foster ME, Lari J. Long-term results of surgery for childhood achalasia. J Pediatr Surg. 1997;32:432-4.

12. Rodrigues JJ G, Sakano IA, Raia AA. Cirurgia do esôfago. In: Goffi FG. Técnica Cirúrgica. Bases Anatômicas, Fisiopatológicas e Técnicas da Cirurgia. $4^{a}$ ed. São Paulo: Atheneu; 1996. p. 528-40.

13. Liu JF, Zhang J, Tian ZQ, Wang QZ, Li BQ, Wang FS, et al. Longterm outcome of esophageal myotomy for achalasia. World J Gastroenterol. 2004;10:287-91.

14. Mattioli G, Esposito C, Prato AP, Doldo P, Castagnetti M, Barabino A, et al. Results of the Laparoscopy Heller-Dor procedure for pediatric esophageal achalasia. Surg Endosc. 2003;17: 1650-2.
Corresponding author:

Fabíola Pollachi

Rua Juréia, 916/133

CEP 04140-110 - São Paulo, SP

Brazil

Phone: +55 (11) 9620.3811/5587.4539

E-mail: fpoll@hotmail.com 